\title{
Acute mycophenolate overdose: case series and systematic literature analysis
}

\author{
Ceschi, Alessandro ; Gregoriano, Claudia ; Rauber-Lüthy, Christine ; Kupferschmidt, Hugo ; Banner,
} Nicholas R ; Krähenbühl, Stephan ; Taegtmeyer, Anne B

\begin{abstract}
Background: Literature regarding acute human toxicity of mycophenolate mofetil (MMF) and enteric-coated mycophenolate sodium (EC-MPS) is limited. Objectives: Our objectives were to describe all cases of overdose with MMF or EC-MPS reported to the Swiss Toxicological Information Centre (STIC) or in the literature between 1995 and 2013. Therefore, we performed an observational case-series and systematic literature search to determine circumstances, magnitude, management and outcome of overdose with MMF or EC-MPS. Results: Of 152,762 reports to STIC, 15 (7 pediatric) involved overdose with MMF ( $\mathrm{n}=13)$ or EC-MPS $(\mathrm{n}=2)$. Three cases from other centers were identified from a systematic literature search. The magnitude of overdose ranged from 1.2 to 16.7 (median 2.9) times usual dose. Six $(33 \%)$ MMF overdoses had attributable symptoms, which included abdominal pain, vomiting, headache and dizziness. The majority of findings were minor, although a 9-fold MMF overdose caused hypotension $8 \mathrm{~h}$ after ingestion and a 12.5-fold overdose caused leukopenia after 5 days. Symptoms did not occur in patients who took 2.5 times or less of their usual MMF dose. Gastrointestinal decontamination measures with activated charcoal were undertaken in one-third of cases. Conclusions: Acute MMF and EC-MPS overdoses had a favorable outcome in all cases reported to STIC and in the literature.
\end{abstract}

DOI: https://doi.org/10.1517/14740338.2014.903032

Posted at the Zurich Open Repository and Archive, University of Zurich

ZORA URL: https://doi.org/10.5167/uzh-94815

Journal Article

Accepted Version

Originally published at:

Ceschi, Alessandro; Gregoriano, Claudia; Rauber-Lüthy, Christine; Kupferschmidt, Hugo; Banner, Nicholas R; Krähenbühl, Stephan; Taegtmeyer, Anne B (2014). Acute mycophenolate overdose: case series and systematic literature analysis. Expert Opinion on Drug Safety, 13(5):525-534.

DOI: https://doi.org/10.1517/14740338.2014.903032 
Acute mycophenolate overdose: case series and systematic literature analysis
A. Ceschi ${ }^{1,2^{*}}$, C. Gregoriano ${ }^{3,} 4^{*}$, C. Rauber-Lüthy ${ }^{1}$, H. Kupferschmidt ${ }^{1}$, N.R. Banner ${ }^{5,} 6$, S.
Krähenbühl $^{3}$, A.B. Taegtmeyer ${ }^{3}$
* contributed equally

${ }^{1}$ Swiss Toxicological Information Centre, Associated Institute of the University of Zurich, Zurich, Switzerland

${ }^{2}$ Department of Clinical Pharmacology and Toxicology, University Hospital Zurich, Zurich, Switzerland

${ }^{3}$ Division of Clinical Pharmacology and Toxicology, University and University Hospital Basel, Basel, Switzerland

${ }^{4}$ University Clinic of Internal Medicine, Kantonsspital Baselland Liestal, Liestal, Switzerland

${ }^{5}$ The Royal Brompton and Harefield NHS Foundation Trust, Harefield Hospital, Harefield, Middlesex, United Kingdom

${ }^{6}$ National Heart and Lung Institute and Institute of Cardiovascular Medicine and Research, Imperial College, London, United Kingdom

Corresponding author:

Alessandro Ceschi, MD

Head, Division of Science

Swiss Toxicological Information Centre

Associated Institute of the University of Zurich

Freiestrasse 16

$\mathrm{CH}-8032$ Zurich

Tel: +41-44-634-1034

Fax: +41-44-252-8833

e-mail: Alessandro.Ceschi@usz.ch 


\begin{abstract}
Objective: Literature regarding acute human toxicity of mycophenolate mofetil (MMF) and enteric-coated mycophenolate sodium (EC-MPS) is limited. Our objectives were to describe all cases of overdose with MMF or EC-MPS reported to the Swiss Toxicological Information Centre (STIC) or in the literature between 1995-2013.
\end{abstract}

Research design and methods: Observational case-series and systematic literature search to determine circumstances, magnitude, management and outcome of overdose with MMF or EC-MPS.

Results: Of 152,762 reports to STIC, 15 (7 paediatric) involved overdose with MMF $(n=13)$ or EC-MPS $(n=2)$. Three cases from other centres were identified from a systematic literature search. The magnitude of overdose ranged from 1.2 to 16.7 (median 2.9) times usual dose. Six (33\%) MMF overdoses had attributable symptoms which included abdominal pain, vomiting, headache and dizziness. The majority of findings were minor, although a 9-fold MMF overdose caused hypotension $8 \mathrm{~h}$ after ingestion and a 12.5 -fold overdose caused leukopenia after 5 days. Symptoms did not occurr in patients who took 2.5-times or less of their usual MMF dose. Gastrointestinal decontamination measures with activated charcoal were undertaken in one third of cases.

Conclusions: Acute MMF and EC-MPS overdoses had a favourable outcome in all cases reported to STIC and in the literature.

Key words: Human toxicity, mycophenolate mofetil, mycophenolic acid, enteric-coated mycophenolate sodium, overdose. 


\section{Introduction}

Mycophenolate mofetil (MMF) and enteric-coated mycophenolate sodium (EC-MPS) are selective immunosuppressants used in combination with other immunosuppressive agents for the prevention of acute rejection after solid organ transplantation in adults and children ${ }^{1}$ ${ }^{2}$. They are also used in the treatment of several autoimmune diseases ${ }^{3}$. Compared to the frequently prescribed thiopurines, these are newer active substances which have been approved for use in Switzerland since 1995 (MMF) and 2002 (EC-MPS). MMF is the prodrug and EC-MPS the sodium salt of the active substance mycophenolic acid (MPA). The term MPA will be used when discussing toxicity because it is the active form of both drugs.

MPA is a selective, reversible inhibitor of inosine monophosphate dehydrogenase (IMPDH) ${ }^{4}$. IMPDH is the rate-limiting enzyme in the synthesis of guanosine nucleotides. As T- and Blymphocyte-proliferation is entirely dependent on the de novo synthesis of guanosine (unlike other cell types which can utilise guanosine from a salvage pathway), MPA exerts a much more potent cytostatic effect on lymphocytes than on other cell types ${ }^{4}$. The most frequently reported side effects of MPA are gastrointestinal disturbances, which are dose-dependent and include nausea, diarrhoea, anorexia, abdominal cramps and vomiting. Reversible, doserelated haematological effects such as anaemia, leukopenia neutropenia and thrombocytopenia are also observed ${ }^{5}$. The long term therapeutic use of MMF and EC-MPS appears to be safe and not to show any serious consequences ${ }^{6,7}$. MMF is rapidly absorbed and de-esterified to MPA after oral administration; the absolute bioavailability of MPA is $94 \%$. Intravenous administration of MMF leads to a comparable exposure as observed after oral administration of the same dose ${ }^{1}$. EC-MPS is readily absorbed in the small intestine because its enteric coating dissolves at $\mathrm{pH} 5.5$. The ingestion of equimolar doses of MMF and EC-MPS lead to similar peak concentrations and exposure, and the degree of IMPDH inhibition is also comparable. The intra- and interpatient pharmacokinetic variability is high for both MPA formulations ${ }^{8,9}$.

The majority of MPA is metabolized in the liver by UDP-glycuronosyltransferases [UGT] in a phase II glucuronidation process. MPA is converted to a major 7-O-glucuronide metabolite 
(MPAG) which is pharmacologically inactive ${ }^{10}$. MPAG is excreted into bile and therefore back into the bowel where gut bacteria can deglucuronidate it to reform MPA which is reabsorbed. This enterohepatic recirculation results in a 'second peak' approximately $6-8$ hours after ingestion, which could add to or prolong toxicity. In addition to MPAG, two other metabolites, MPA-acyl-glucoronide (AcMPAG) and MPA-phenyl-glucoside (glucoside-MPA), are observed in plasma ${ }^{11}$. In vitro analysis has shown that AcMPAG is pharmacologically active and is likely responsible for the gastrointestinal toxicity of MPA ${ }^{12}$. Approximately $93 \%$ of the glucuronide metabolites are renally excreted ${ }^{10}$.

Patients taking MMF or EC-MPS may be intentionally or accidentally exposed to acute overdose. In addition, their household contacts may have access to these drugs and also be at risk of overdose. The purpose of this study was to investigate the circumstances, management and outcomes of overdoses with MMF or EC-MPS using data reported to a single national poison centre and data reported in the literature during an 18-year period.

\section{Methods}

\subsection{Study design}

Specific ethics approval was not required for this observational study due to the nature of the study design in accordance with the regulations of the cantonal ethics committee Zurich, Switzerland which also stated that anonymised data generated during patient care can be used retrospectively for research purposes without obtaining written consent. We performed a retrospective review of all acute overdoses involving MMF or EC-MPS in adults and children ( $<16$ years) either alone or in combination with other drugs that had been reported to the Swiss Toxicological Information Centre (STIC) between April 1995 and August 2013 (for further details, see the online supplement). Cases were assigned an internal identification number so neither the patients or caregivers nor the reporting professionals could be identified by the investigators. 
A systematic literature-search was performed in MEDLINE and EMBASE from 1995 to 2013 using the terms 'mycophenolate` (which encompases mycophenolate sodium, mycophenolic acid and mycophenolate mofetil) and 'overdose'. Furthermore the relevant product information ${ }^{1,2}$ and guidance on the management of acute overdose ${ }^{13,14}$ were examined.

\subsection{Circumstances and symptoms of overdose}

The circumstances of overdose were categorised into 'suicidal' for cases of intentional overdose, 'domestic' for cases of accidental overdose in the home and 'iatrogenic' for those due to a prescribing or administration error by a healthcare professional. The severity of symptoms were graded in accordance with the Poisoning Severity Score (PSS) as 'minor', for mild, transient and spontaneously resolving symptoms/signs; 'moderate', if at least one pronounced or prolonged symptom/sign was recorded; 'severe', if at least one severe or lifethreatening symptom/sign was observed, or 'fatal', if the overdose was the recorded cause of death ${ }^{14}$. Cases were assessed for association between symptoms and the MMF or ECMPS overdose by an expert panel including a senior clinical toxicologist and a clinical pharmacologist, both with additional qualifications in general internal medicine, using the World Health Organisation Uppsala Monitoring Centre (WHO-UMC) standardised case causality assessment criteria originally developed for the assessment of adverse drug reactions ${ }^{16}$. The magnitude of overdose, co-ingestion of other medication (in patients with multiple drug overdose or taking other drugs in the therapeutic dose range) and comorbidities were taken into consideration. Associations were classified as 'certain`, 'likely', ‘possible` and 'unlikely` (Table S1 online supplement).

\subsection{Data analysis}

Descriptive statistics were used to analyse grouped data. Overdoses in $\mathrm{mg} / \mathrm{kg}$ for adults and in $\mathrm{mg} / \mathrm{m}^{2}$ in children were compared with usual therapeutic doses by determining the multiple of the subject's usual therapeutic dose (dose ingested or dose received/usual dose) as 
performed in our previous studies ${ }^{17,18}$. In subjects who did not normally receive MMF or ECMPS or in cases where the usual maintenance dose was not known, the magnitude of overdose above the maximum licensed dose was determined. In Switzerland the maximum licensed oral MMF dose for treatment of adults is $2-3 \mathrm{~g} /$ day, depending on indication. The recommended dose for oral MMF in children and adolescents is $600 \mathrm{mg} / \mathrm{m}^{2}$ body surface area twice daily up to a maximal daily dose of $2 \mathrm{~g}$. The maximum licensed dose for intravenous MMF is $1 \mathrm{~g} / 12 \mathrm{~h}^{1}$. The infusion should be administered slowly over a period of 2 hours. The recommended dose of EC-MPS for adults is $720 \mathrm{mg}$ twice daily ${ }^{2}$. EC-MPS is not licensed for children in Switzerland ${ }^{2}$, but is licensed in other countries such as the United States of America ${ }^{19}$. The Food and Drug Administration licensed dose is $400 \mathrm{mg} / \mathrm{m}^{2}$ twice daily for renal transplant rejection prophylaxis in children aged $5-16$ years ${ }^{19}$. Missing data regarding patient weight was computed as detailed in the online supplement. The drug half-life was calculated according to the standard pharmacokinetic equation given in the online supplement in cases where serial drug concentration measurements had been performed

\section{Results}

\subsection{Cases}

A total of 152,762 reports by healthcare professionals to the STIC of confirmed or suspected overdose with any substance occurred during the study period. Of these, 13 were with MMF and two with EC-MPS (7 paediatric cases). In 14 cases the route of administration was oral (tablets, capsules or oral solution) while in one case MMF was administrated as an intravenous solution. The majority of the cases were reported by hospital doctors $(n=13)$, one case by another doctor and one case by a caregiver. Demographic characteristics, circumstances of overdose, the magnitude of overdose and the availability of follow-up data are given in Table 1 along with the details of three cases published in the literature ${ }^{20-22}$. Adults and children are presented separately. Table 2 shows the details of each individual case. For the cases reported to STIC, the mean age of adult cases was 31.5 years (range 
19-50). Six (40\%) cases involved patients already receiving immunosuppression, eight cases (53\%) did not normally receive immunosuppression and in one case the underlying diagnosis was not known. Six $(86 \%)$ of the seven accidental domestic overdoses were in children (age range 1.5-9 years). Four of these were living with parents or siblings undergoing treatment with MMF or EC-MPS. One case of dosing error in the home (patient 13) involved a mother administering $2 \mathrm{ml}$ of dry MMF powder to her child rather than reconstituting the powder with water and administering $2 \mathrm{ml}$ of the resulting solution as per instructions. The cases labelled ' $15^{\prime}$ ' ' $^{\prime} 16^{\prime}$ and ' $17^{`}$ in tables 2 and 3 are those which have been published previously ${ }^{20-22}$. All three were females with an underlying diagnosis requiring immunosuppression. In two cases (patients 15 and 16) the overdose was deliberate (with suicidal intent) while in patient 17 the overdose was caused iatrogenically during a drug switch from twice daily MMF to once daily EC-MPS in an attempt to improve adherence (although EC-MPS is licensed to be given every 12 hours $^{2}$ ). Therapeutic drug monitoring showed a significantly higher drug exposure after once daily $1.44 \mathrm{~g}$ enteric-coated EC-MPS than under the previous MMF $1 \mathrm{~g}$ twice-daily dose ${ }^{22}$. The dose of EC-MPS was subsequently reduced to $900 \mathrm{mg}$ once daily.

\subsection{Magnitude of overdose}

Table 3 shows the magnitude of overdose in relation to the patient's usual maintenance dose or - for treatment-naïve individuals - in relation to the maximum licensed dose. Two of the exposures were retrospectively found to be equal to or less than the maximum licensed dose and were a case of accidental ingestion by a child (patient 2) and a case of iatrogenic error (patient 18). These two cases were considered `accidental exposures within the therapeutic range rather than 'overdose` and were not included in the analysis of symptom development in order to accurately reflect the effect of true overdose. The overdoses ranged from 1.2 to 16.7 times the usual or maximum licensed dose (Table 2). 
Drug concentration measurements were only available in one case reported to STIC. Patient 7 (a 12-year-old female) had a plasma MPA concentration of $0.5 \mathrm{mg} / \mathrm{l}$ approximately 24 hours after overdose with $5 \mathrm{~g}$ MMF. Being a drug which is administered twice daily, no data regarding expected plasma concentrations 24 hours after a single administration in children exist, so interpretation of this result was not possible. However, by way of comparison, the therapeutic range of trough MPA concentrations after liver transplantation is $1-3.5 \mathrm{mg} / \mathrm{L}$ and $2.5-4.5 \mathrm{mg} / \mathrm{l}$ after heart transplantation ${ }^{23}$.

Plasma drug concentration measurements were available for all the published cases. The measured serial MPA concentrations after ingestion of $10 \mathrm{~g} \mathrm{MMF}$ (patient 15) were $44.1 \mathrm{mg} / \mathrm{l}$ ( 5 hours after ingestion), $5.6 \mathrm{mg} / \mathrm{l}$ (10 hours after ingestion) and $0.3 \mathrm{mg} / \mathrm{l}$ ( 21 hours after ingestion) ${ }^{21}$. Serial plasma MPA concentrations after ingestion of $25 \mathrm{~g}$ (patient 16) were 37.2 mg/l (20 hours after ingestion), $1.1 \mathrm{mg} / \mathrm{l}$ (24 hours after ingestion), $0.4 \mathrm{mg} / \mathrm{l}$ (48 hours after ingestion) and $0.1 \mathrm{mg} / \mathrm{l}\left(72\right.$ hours after ingestion) ${ }^{20}$. Patient 17 had a maximum MPA concentration of $45.1 \mathrm{mg} / \mathrm{l}$ three hours after ingestion of $1440 \mathrm{mg}$ EC-MPS ${ }^{22}$.

\subsection{Pharmacokinetic calculations}

The half-lives of MPA were $2.6 \mathrm{~h}$ and $6.1 \mathrm{~h}$ in patients 15 and 16 , respectively. By reading raw data points off the concentration-time graph in the publication describing patient $17^{22}$, an estimated half-life of $7.4 \mathrm{~h}$ after ingestion of EC-MPS could be calculated.

\subsection{Outcomes}

The outcomes of overdose are given in detail in Table 3. In the cases reported to STIC, MMF overdoses were judged to have caused or contributed to symptoms or abnormal clinical findings in 4 of the 13 true overdose cases. All symptoms attributable to mycophenolate were minor and included abdominal pain $(n=2)$, vomiting $(n=2)$, headache $(n=2)$ and dizziness $(n=1)$. One patient (patient 3 ) experienced a fall in blood pressure from 113/68 $\mathrm{mmHg}$ on admission 4 hours after overdose to $90 / 40 \mathrm{mmHg} 8$ hours after overdose. The hypotensive episode lasted 2 hours and responded to intravenous fluid therapy. It was 
classified as a moderate sign. No changes in blood count were observed in the cases reported to STIC; one case reported in the literature (patient 16) developed moderate leukopenia with a nadir of $2040 / \mathrm{mm}^{3} 5$ days after overdose (patient's usual range 3500 to $5000 / \mathrm{mm}^{3}$, normal range $5000-10000 / \mathrm{mm}^{3}$ ). None of the EC-MPS overdoses reported to STIC or in the literature caused symptoms, however these overdoses were all modest in size (1.2 - 2 times usual or maximum licensed dose). Overall, patients who developed symptoms after overdose had been exposed to a mean of $8.5+/-4.7$ times their usual or maximum licensed dose compared to asymptomatic patients who were exposed to $2.4+/-1.7$ times their usual or maximum licensed dose. No patient taking 2.5 times or less than their usual or maximum licensed dose developed symptoms.

\subsection{Management}

Of the cases reported to STIC, three patients were discharged home on the same day of presentation either immediately after initial evaluation in the emergency room or after a brief period of observation. All three were asymptomatic. Five patients were admitted to acute medical wards (one subsequently self-discharged against medical advice). Of those hospitalised, median length of hospital stay was 1 day (range 1-2). Hospitalisation status was unknown in 4 patients. Care was transferred from acute medical to psychiatric in-patient services in one case.

The recommendation for use and subsequent use of decontamination measures is shown in Table 3. Of the cases reported to STIC, decontamination measures with activated charcoal were performed in four cases; all of which were children who presented to emergency services within one hour of ingestion. In three patients it is not known if any action was performed. However, the administration of activated charcoal was recommended in two of these cases. Decontamination with activated charcoal was also performed in the paediatric patient who experienced accidental exposure within the therapeutic range (patient 2). For the patients who did not receive activated charcoal, median time until presentation to emergency 
services was two hours (range $<1-5.5 \mathrm{~h}$ ). A follow-up blood count was performed in 1 case of MMF overdose (patient 7, one day after overdose) with a normal result.

Among the cases reported in the literature, patient 15 presented to the emergency room 4 hours after ingestion and was treated with activated charcoal and supportive care (promethazine for nausea and intravenous fluids). She was hospitalized, but the length of stay was not specified ${ }^{21}$. Patient 16 presented to the emergency room approximately 10 hours after ingestion, where she was treated with activated charcoal and oral cholestyramine $(8 \mathrm{~g})$ every 8 hours. She was hospitalized for 6 days ${ }^{20}$. Patient 17 underwent a dosereduction of her maintenance EC-MPS.

\subsection{Effect of decontamination}

All four patients who received activated charcoal after overdose remained asymptomatic compared to four of the eight $(50 \%)$ who did not undergo gastrointestinal decontamination among the cases reported to STIC. The mean multiple above patients' usual or maximum licensed dose of MMF or EC-MPS was higher in untreated patients $(4.1 \pm 2.9)$ than in treated patients $(1.2 \pm 0.3)$. No adverse effects resulting from treatment with activated charcoal were reported. Patient 16 who was treated with oral cholestyramine in addition to activated charcoal developed mild anorexia, headache and moderate leukopenia, despite the high ingested dose ${ }^{20}$.

\section{Discussion}

This case-series analysis of MMF and EC-MPS overdoses reported to a single national poison centre between 1995 and 2013 adds a further 15 cases to the three case reports currently published in the medical literature. Acute overdoses of these substances are rare and seem to be generally well tolerated. As the experience with acute overdose of MMF and EC-MPS in humans is very limited, the data presented here gives an insight into the patterns, management strategies and outcomes of MMF and EC-MPS overdoses. 


\subsection{Circumstances of overdose}

A number of important issues emerge when examining the circumstances of overdose with MMF or EC-MPS. In contrast to our previous findings for calcineurin inhibitor $(\mathrm{CNI})$ overdose where iatrogenic errors accounted for $46 \%$ of the reported cases ${ }^{17}$, iatrogenic dosing errors only accounted for $2(11 \%)$ of all the cases presented here. This may be due to the fact that liquid formulations (intravenous or oral), which accounted for almost three quarters of the $\mathrm{CNI}$ iatrogenic overdoses, are much less commonly used for the administration of MMF, and EC-MPS is only available in tablet form. Nonetheless, extra vigilance should be paid when dealing with such drug forms, as mistakes can easily be made when procedures are unfamiliar. This also holds true for people who take responsibility for drug administration in the home, in particular parents, who should be clearly instructed how to reconstitute powder to make an oral solution. The majority $(75 \%)$ of MMF and EC-MPS overdoses in adults were with suicidal intent. This is in accordance with the proportion reported in the medical literature to date where 2 of the 3 reports were cases of suicidal intent. Both patients suffered from a chronic condition requiring immunosuppression ${ }^{20,21}$. The number of accidental overdoses in the home was also higher than for the previously reported CNI and thiopurine overdose series: $47 \%$ in the current series compared to $11 \%$ among $\mathrm{CNI}$ overdoses and $30 \%$ among thiopurine overdoses ${ }^{17,18}$ which further emphasises the need to repeatedly instruct patients to keep medicines out of reach of unintended recipients.

\subsection{Magnitude and Outcomes}

MMF overdose was associated with symptoms in a dose-dependent manner. These were mainly headache or gastrointestinal-related and were minor in nature. Gastrointestinal symptoms such as nausea, vomiting, diarrhoea or cramping are also the most common adverse effects of these active substances when given in therapeutic doses ${ }^{1}$. One case of haemodynamically significant hypotension occurred 8 hours after ingestion. This is in 
keeping with the enterohepatic recycling of MPAG, which means a second peak MPA concentration is reached approximately $6-12$ hours after oral ingestion ${ }^{1}$. The mechanism underlying mycophenolate-induced hypotension is not known.

Patient 16 was found to be leukopenic 14 hours after overdose ${ }^{20}$. Whether this was due to the overdose is not certain as the leukocyte count had not been measured during the days and weeks immediately preceding the overdose and she was also receiving quetiapine, an agent known to be frequently (> 10\%) associated with leukopenia ${ }^{24}$. In our opinion, the mechanism whereby MPA causes leukopenia (disruption of de novo guanosine synthesis) is unlikely to lead to such a rapid decline in the leukocyte count and it is likely that the leukocyte count was already decreased prior to overdose in this particular case. After overdose in this patient, the leukocyte count continued to fall, reaching a nadir 5 days later. The oral acute lethal dose 50 (LD50) for MMF in rats is $352 \mathrm{mg} / \mathrm{kg}$, in mice $1000 \mathrm{mg} / \mathrm{kg}$ and $>6000 \mathrm{mg} / \mathrm{kg}$ in rabbits, indicating a species-dependence of the lethal dose ${ }^{25}$. The maximum overdose in this current series was $448 \mathrm{mg} / \mathrm{kg}$.

\subsection{Pharmacokinetics:}

The apparent half-life of MPA is 16 - 18 hours; an accurate determination of MPA`s half-life is hampered by MPAG's enterohepatic recirculation ${ }^{1,10}$. In the literature cases studied here, terminal half-life was $2.6 \mathrm{~h}$ in a case where activated charcoal was given 4 hours after overdose (Patient 15), $6.1 \mathrm{~h}$ in a case where activated charcoal and cholestyramine were given $10 \mathrm{~h}$ after overdose (patient 16) and $7.4 \mathrm{~h}$ (patient 17). These data imply a reduction in the MPA half-life when activated charcoal alone or in combination with cholestyramine is used in the management of MMF overdose. A study of liver transplant patients, however, also found a shorter terminal disposition half-life (mean $4.54 \pm 1.19 \mathrm{~h}$ ) than the pharmacokinetic studies in healthy volunteers ${ }^{26,10}$. Unlike concomitant cyclosporin, tacrolimus (the calcineurin inhibitor used in this study) is not known to disrupt the enterohepatic cycling of MPAG so this cannot explain the shorter half-life seen ${ }^{1}$. 


\subsection{Management}

Due to the rarity of MMF and EC-MPS overdoses, it is not possible to perform randomised controlled trials to determine the optimal management, and examination of case data such as that presented here is therefore important.

Patients who ingested 2.5 times or less than their usual or the maximum recommended MMF or EC-MPS dose did not develop symptoms and medical treatment or hospitalisation of such cases (other than for psychiatric reasons) does not appear to be indicated. Similarly, in our opinion, activated charcoal should only be used in cases of definite overdose as its administration is not without risk ${ }^{27}$. The fact that activated charcoal was only administrated to children in the cases reported to STIC most likely reflects their earlier presentation to emergency services (all within $1 \mathrm{~h}-$ table 3 ).

In this case-series, patients who received activated charcoal developed fewer symptoms. However, it is not possible to conclude a definite beneficial effect of activated charcoal after mycophenolate overdose as these patients had presented earlier and had ingested, on average, smaller doses than patients who did not receive activated charcoal.

The Swiss product information for MMF and EC-MPS do not recommend the use of activated charcoal after overdose ${ }^{1,2}$. Other sources, however, recommend that activated charcoal be used 'early' in overdose of these drugs ${ }^{14}$ and that it is most effective when administered within $1 \mathrm{~h}$ of overdose ${ }^{13}$. On the basis of their chemical properties, a good adsorption of MMF and EC-MPS onto activated charcoal is to be expected. MMF and EC-MPS have molecular weights of 433 and 320 Dalton respectively ${ }^{28}$, which means that they are readily adsorbed into the $10-20 \AA$-sized charcoal pores ${ }^{29}$. As the absolute bioavailability of MPA after oral administration of MMF is high (94\%) and MPA reaches its peak plasma concentration between 1.5 and 2 hours after ingestion ${ }^{1}$, it is reasonable to expect that activated charcoal is beneficial when administered within 2 hours after overdose. One can also speculate that charcoal administered later than 2 hours would still be of benefit because it could reduce the 'second peak' resulting from reabsorption of MPAG excreted in the bile, 
thereby minimising the effect of the enterohepatic recirculation. In the cases published by Bebarta and Wu, activated charcoal was given 4 and 10 hours after overdose, respectively ${ }^{21}$ ${ }^{20}$, and the half-lives were found to be less than expected. This provides some evidence to support the use of activated charcoal beyond 2 hours after overdose in MMF and EC-MPS overdose.

The administration of bile acid sequestrants, such as cholestyramine, is recommended in the treatment of acute overdose with MMF or EC-MPS in the Swiss product information ${ }^{1,2}$ but not specifically by the widely consulted Poisindex ${ }^{\circledR}$ Database ${ }^{13}$. By binding parent and glucuronidated drug that is excreted in the bile, cholestyramine prevents their reabsorption and deglucuronidation, respectively and thereby interrupts the enterohepatic recycling of MPAG ${ }^{10}$. Patient 16 who ingested $25 \mathrm{~g}$ of MMF in suicidal intent, was treated with oral cholestyramine (8 $\mathrm{g}$ three times daily for three days) commencing $14 \mathrm{~h}$ after overdose and did not developed hypotension or other symptoms in the time periods when a 'second peak' might have been expected to occur ${ }^{20}$. Also of note in this case was the rapid decrease in MPA concentrations after commencement of cholestyramine from $37.2 \mathrm{mg} / \mathrm{l}$ at 20 hours to $1.1 \mathrm{mg} / \mathrm{l}$ just 4 hours later.

Interestingly the STIC case in which hypotension was observed eight hours after overdose had neither been treated with activated charcoal nor with cholestyramine. This case also illustrates the point that it would seem prudent to monitor patients who have ingested large MMF or EC-MPS doses or who are symptomatic at presentation for at least $12-24$ hours after overdose due to the possible risk of delayed hypotension.

\subsection{Limitations}

Our study has a number of limitations, primarily related to the small sample size. Larger series of MMF or EC-MPS overdoses have, however, not been published. It is likely that our data did not capture all cases of overdose which occurred in the referral population. Our data are also incomplete, which is the nature of retrospective studies using poison centre data ${ }^{30}$. Unfortunately, a number of cases were lost to follow-up despite repeated efforts of the STIC 
to contact the treating physicians in the days after presentation. In some cases follow-up took place sooner than 7 days after overdose which is the time point when maximum bone marrow suppression after ingestion of a bone marrow suppressing agent such as MMF and EC-MPS might be expected. A further limitation is the paucity of data regarding blood or plasma concentrations which would have allowed stronger conclusions regarding pharmacokinetic-pharmacodynamic relationships and the effect of decontamination on drug absorption to be drawn. However, such data are extremely difficult to obtain as therapeutic drug monitoring for MMF and EC-MPS are not routinely performed.

\section{Conclusion}

Acute MMF and EC-MPS overdoses occurred commonly in the context of suicide attempt among adults and in the context of accidental poising in the home among children. The acute overdoses were well tolerated, with the exception of a 9-fold MMF overdose which was associated with hypotension eight hours after ingestion possibly reflecting the enterohepatic circulation of MPAG and a case of moderate leukopenia 5 days after a 12.5-fold overdose. More data are needed to determine the effect of decontamination with activated charcoal and cholestyramine. MMF and EC-MPS overdose patterns, outcomes and management require further study and physicians should be encouraged to actively follow-up and report the cases in which they are involved.

\section{Acknowledgements}

Funding was through internal funds of University and University Hospital Basel and STIC. SK is supported by grants from the Swiss National Science Foundation (SNF31003A-132992). The authors declare that they have no conflict of interest. 


\section{Supporting Information}

Additional Supporting Information may be found in the online version of this article.

- Supplemental Methods

- Supplemental Table S1: World Health Organisation Uppsala Monitoring Centre (WHO-UMC) causality categories ${ }^{16}$ 


\section{References}

1. Cellcept ${ }^{\circledR}$. Product information. Roche Pharma (Schweiz) AG, Reihnach, Switzerland 2013.

2. Myfortic®. Product information. Novartis Pharma Schweiz AG, Risch, Switzerland 2012.

3. Abd Rahman AN, Tett SE, Staatz CE. Clinical pharmacokinetics and pharmacodynamics of mycophenolate in patients with autoimmune disease. Clinical Pharmacokinetics 2013;52:303-31.

4. Allison AC, Eugui EM. Mycophenolate mofetil and its mechanisms of action. Immunopharmacology 2000;47:85-118.

5. Kitchin JE, Pomeranz MK, Pak G, et al. Rediscovering mycophenolic acid: a review of its mechanism, side effects, and potential uses. Journal of the American Academy of Dermatology 1997;37:445-9.

6. Ensley RD, Bristow MR, Olsen SL, et al. The use of mycophenolate mofetil (RS-61443) in human heart transplant recipients. Transplantation 1993;56:75-82.

7. Goldblum R. Therapy of rheumatoid arthritis with mycophenolate mofetil. Clinical and experimental rheumatology 1993;11 Suppl 8:S117-9.

8. Budde K, Dürr M, Liefeldt L, Neumayer HH, Glander P. Enteric-coated mycophenolate sodium. Expert Opin Drug Saf. 2010;9:981-94.

- This is a seminal review about the clinical pharmacolgy of enteric-coated mycophenolate sodium

9. Filler G, Foster J, Berard R, Mai I, Lepage N. Age-dependency of mycophenolate mofetil dosing in combination with tacrolimus after pediatric renal transplantation. Transplant Proc. 2004;36:1327-31.

10. Bullingham RE, Nicholls AJ, Kamm BR. Clinical pharmacokinetics of mycophenolate mofetil. Clinical Pharmacokinetics 1998;34:429-55.

- This is a seminal review about the clinical pharmacolgy of mycophenolate mofetil

11. Schutz E, Shipkova M, Armstrong VW, et al. Therapeutic drug monitoring of mycophenolic acid: comparison of HPLC and immunoassay reveals new MPA metabolites. Transplantation Proceedings 1998;30:1185-7.

12. Wieland E, Shipkova M, Schellhaas U, et al. Induction of cytokine release by the acyl glucuronide of mycophenolic acid: a link to side effects? Clinical Biochemistry 2000;33:107-13.

13. Mycophenolate In: Poisindex® [Internet database]. Greenwood Village, Colo: Thomson Reuters (Healthcare) Inc. Updated periodically.

14. Mycophenolate. In: ToxPoints $₫$ Summary [Internet database]. Greenwood Village, Colo: Thomson Reuters (Healthcare) Inc. Updated periodically.

15. Persson HE, Sjoberg GK, Haines JA, et al. Poisoning severity score. Grading of acute poisoning. Journal of Toxicology Clinical Toxicology 1998;36:205-13.

16. The Uppsala Monitoring Centre, Uppsala, Sweden. The use of the WHO-UMC system for standardised case causality assessment. Available at: whoumc.org/Graphics/24734.pdf [Last accessed 10 January 2014].

17. Ceschi A, Rauber-Luthy $\mathrm{C}$, Kupferschmidt $\mathrm{H}$, et al. Acute calcineurin inhibitor overdose: analysis of cases reported to a national poison center between 1995 and 2011. American Journal of Transplantation. 2013;13:786-95.

- This report presents data from the same poisons centre as the current work 18. Gregoriano C, Ceschi A, Rauber-Lüthy C, et al. Acute thiopurine overdose: analysis of reports to a national poison centre 1995 - 2013. PLoS One 2014;9:e86930. 
- This report presents data from the same poisons centre as the current work 19. Myfortic $\circledast$. Product information. Novartis Pharmaceuticals Corporation, East Hanover, New Jersey, USA 2013.

20. Wu SW, Chang HR, Lai YR, Lian JD. Non-life-threatening leukopenia in a renal transplant recipient with acute overdose of mycophenolate mofetil. Transplantation Proceedings 2008;40:3770-1.

- This is one of the case-reports reviewed

21. Bebarta VS, Heard K, Nadelson C. Lack of toxic effects following acute overdose of cellcept (mycophenolate mofetil). Journal of Toxicology Clinical Toxicology 2004;42:917-9.

- This is one of the case-reports reviewed

22. Filler G, Lathia A, LeBlanc C, Christians U. Unexpectedly high exposure to enteric-coated mycophenolate sodium upon once-daily dosing. Pediatric Nephrology 2006;21:1206-8.

- This is one of the case-reports reviewed

23. van Gelder T, Le Meur Y, Shaw LM, Oellerich M, et al. Therapeutic drug monitoring of mycophenolate mofetil in transplantation. Therapeutic Drug Monitoring 2006;28:145-54.

24. Seroquel. Product Information AstraZeneca AG, Zug, Switzerland 2012.

25. Wishart DS, Knox C, Guo AC, et al. DrugBank: a knowledgebase for drugs, drug actions and drug targets. Nucleic Acids Research 2008;36:D901-6.

26. Jain $A$, Venkataramanan $R$, Kwong $T$, et al. Pharmacokinetics of mycophenolic acid in liver transplant patients after intravenous and oral administration of mycophenolate mofetil. Liver Transplantation 2007;13:791-6. 27. Mauro LS, Nawarskas JJ, Maruo VF. Misadventures with activated charcoal and recommendations for safe use. Ann Pharmacother 1994;28:915-24.

28. The PubChem Project. Available at: http://pubchem.ncbi.nlm.nih.gov/ [Last accessed 13th December 2013].

29. Howland MA. Activated Charcoal. In: Nelson LS, Lewin NA, Howland MA, Hoffman RS, Goldfrank LR, Flomenbaum NE, editors. Goldfrank`s Toxicologic Emergencies Ninth Edition. New York: McGraw-Hill Medical. pp. 108-114, 2010. 30. Hoffman RS. Understanding the limitations of retrospective analyses of poison center data. Clinical Toxicology 2007;45:943-5. 